\title{
Enigmas in the making
}

lan Stewart

Collected Works of A. M. Turing. 3 Volumes*. By A. M. Turing. North-Holland: 1992/1993. Distributed by Elsevier.

ALAN Mathison Turing is one of the great original minds of the twentieth century, a founding father of the electronic computer whose name lives on in such concepts as the Turing machine, a simple model for the computational process, and the Turing test, a method he proposed for deciding whether a machine is engaged in genuine thought. This handsome edition of his collected works - of which a fourth volume, Mathematical Logic, has yet to appear - includes much unpublished material, and offers new insights into his interests and his mentality. As well as being a great scientist, Turing was also a tragic figure, a quiet homosexual who was pushed to suicide by a discriminatory judicial system. But it is his scientific work that we encounter here.

Turing published very little: in these three volumes we find one published paper on morphogenesis, eight on pure mathematics and seven on machine intelligence (of which two are technical reports and one is a 1947 lecture that has only recently seen print). Perhaps half of the material consists of drafts of papers and articles left in Turing's archives.

The seminal paper The Chemical Basis of Morphogenesis dates from 1952. Turing aims to model the developing embryo in terms of the diffusion and reaction of chemical substances called morphogens. Among his key ideas is the spontaneous formation of patterns arising from instabilities of the homogeneous state. He anticipates, in this special case, many modern ideas about symmetry-breaking and bifurcation. The specific mechanism that he proposes did not stand up to detailed experimental scrutiny, and has not found much favour among mainstream biologists, especially in an age that is strongly oriented towards the molecular view of biology and the DNA code. But Turing's central idea has been modified by several groups of mathematical biologists to make it more compatible with known biological facts, and there is a growing body of evidence that the development of biological form must involve dynamic, as well as molecular, processes. In this volume we also find unpublished papers on phyllotaxis - the arrangement of leaves on plants.

Turing's work in pure mathematics is

*Volume 1: Pure Mathematics edited by J. L. Britton, pp. 288 , DFL175, $\$ 89.50$ (1992):

Volume 2: Mechanical Intelligence edited by D. C. Ince, pp Volume 2: Mechanical Inte

226, DFL160, $\$ 82$ (1992);
Volume 3: Morphogenesis edited by P. T. Saunders, pp. 160 , DFL170, \$106.50 (1993) focused on three main areas: group theory, analytic number theory and the word problem. A paper of 1938 provides a partial answer to a question of Stanislaw Ulam on the approximation of groups by finite groups; the proof is rather interesting, involving an interplay between the representation theories of fi-

\section{IMAGE UNAVAILABLE FOR COPYRIGHT REASONS}

\section{Alan Turing (1912-1954).}

nite and compact Lie groups. Turing's number-theoretic research centres on the Riemann zeta function, whose analytic properties are important for the distribution of prime numbers. Perhaps the unsolved problem in mathematics is the Riemann hypothesis that all non-real zeros of the zeta function lie on the line 'real part $=1 / 2$ ' in the complex plane. Turing put a lot of effort into devising effective numerical methods for locating these zeros accurately: here we see his interest in computers impinging on other, more ethereal areas. A quite remarkable unpublished manuscript, written jointly (or perhaps not) with S. Skewes, deals with the error term in the prime number theorem. This theorem relates the number $\pi(x)$ of primes less than some bound $x$ to the logarithmic integral li $(x)$. Carl Friedrich Gauss conjectured, and Jacques Hadamard and Charles-Jean de la Vallée Poussin proved, that the ratio $\pi(x) / \operatorname{li}(x)$ tends to 1 as $x$ tends to infinity. Numerical evidence available in the early 1900 s suggested that $\operatorname{li}(x)$ is always greater than $\pi(x)$, but John Edensor Littlewood proved that the difference changes sign arbitrarily infinitely often. Skewes, his student, proved that $\operatorname{li}(x)$ is less than $\pi(x)$ for some $x$ less than the gigantic number $10 \uparrow 10 \uparrow 10 \uparrow 34$ (where $\uparrow$ is exponentiation), on the assumption of the Riemann hypothesis. Skewes and Turing attempt to improve this to $\exp (\exp (661))$, without assuming the Riemann hypothesis. Their method is basically sound but some errors need repairing, and the value of $x$ becomes $\exp (\exp (1236))$, as A. M. Cohen and M. J. E. Mayhew reported in 1965. Their paper is included.

The most important paper here is a poof that the word problem in cancelo lation semigroups is insoluble. The gist of the problem is that an algebraic structure of a particular kind is defined by sequences of symbols, together with transformation rules; we then ask whether an algorithm exists to decide when two symbol strings are equivalent under such transformations. Turing proved that the answer is 'no', by relating the problem to his work on Turing machines. Subsequently, P. S. Novikov proved the insolubility of the word problem for groups, building on Turing's result.

Finally, we have the work on machine intelligence. In a paper of 1948, Turing predicts three important ideas: subroutines, automated software verification and machine learning. The key offering is the 1950 paper in Mind, which begins: "I propose to consider the question, 'Can machines think?'" Turing's proposed test for machine intelligence is hotly debated even today; the original paper is well worth re-reading, although the central issue is more one of definition - influenced by ideology - than of philosophy.

Turing was a deep thinker whose career was hugely disturbed by the Second World War and who in any case felt little urge to publish. Accordingly, the overall effect of his collected writings is rather bitty - an impression not entirely helped by the format of these volumes, with their many prefatory notes, analyses and interpolated explanations. But the sheer power of the man's mind shines through like a beacon. It is a pity that the hypocrisy of a bygone age put a premature end to such a sparkling scientific career; but we should not be too smug about today's more relaxed attitudes when the careers of many equally promising young scientists are being snuffed out, even before they start, by a political system that knows the price of everything and the value of nothing.

Ian Stewart is in the Mathematics Institute, University of Warwick, Warwick, Coventry CV4 7AL, UK. 